\title{
Avaliação do Desempenho e Consumo de Energia de Aplicações Implementadas com OpenMP SIMD
}

\author{
Gustavo L. Tamiosso ${ }^{1}$, Gustavo P. Berned ${ }^{1}$, Marcelo C. Luizelli ${ }^{1}$, \\ Fábio D. Rossi ${ }^{2}$, Jorji Nonaka ${ }^{3}$ e Arthur F. Lorenzon ${ }^{1}$ \\ ${ }^{1}$ Laboratório de Otimização de Sistemas - Universidade Federal do Pampa \\ Campus Alegrete (UNIPAMPA) - Alegrete - RS - Brasil \\ ${ }^{2}$ Instituto Federal Farroupilha - Campus Alegrete - Alegrete - RS - Brasil \\ ${ }^{3}$ RIKEN Center for Computational Science, Japan \\ gustavotamiosso.aluno@unipampa.edu.br
}

\begin{abstract}
Resumo. Explorar o paralelismo tornou-se uma alternativa para reduzir o consumo de energia e melhorar o desempenho de aplicações. Este trabalho tem como objetivo avaliar as estatísticas de desempenho e energia de aplicações implementadas utilizando operações vetoriais. Através da execução de quatro aplicações conhecidas, mostramos que o uso de operações vetoriais pode reduzir o tempo de execução em até $37 \%$ e reduzir o consumo de energia em $35 \%{ }^{1}$
\end{abstract}

\section{Introdução}

A exploração do paralelismo no nível de threads tem sido amplamente utilizada para melhorar o desempenho de processadores com vários núcleos. No entanto as arquiteturas adquiriram a habilidade de realizar instruções de forma vetorial, com o uso de registradores embutidos em sua construção. Esta computação, também conhecida como SIMD (single instruction, multiple data), utiliza unidades vetoriais para acelerar operações aritméticas que são particularmente realizadas sob estruturas de dados vetoriais.

Diferentes otimizações e extensões para compiladores e interfaces de programação paralela (e.g., Open Multiprocessing - OpenMP) têm sido desenvolvidas para facilitar o uso de operações SIMD por parte dos programadores. O OpenMP permite aos programadores explorarem estas instruções através do uso da diretiva \#pragma omp simd, que indica ao compilador quais regiões de código devem ser aplicadas a vetorização. Esta diretiva pode ser utilizada em uma região sequencial ou associada à regiões paralelas (interna a regiões \#pragma omp parallel for). Neste último, cada thread irá explorar o uso de operações vetoriais em seu núcleo de processamento [Chapman et al. 2007].

Sendo assim, este trabalho tem como objetivo principal comparar o desempenho e consumo de energia de aplicações implementada com OpenMP SIMD. Para tanto, nós utilizamos quatro aplicações com diferentes características relacionadas às estruturas de dados utilizadas e operações vetoriais/matriciais. Através da execução das aplicações em um processador AMD Ryzen 9 com suporte a execução de até 24 threads e operações AVX-2 (256-bits), nós mostramos que comparando o melhor resultado obtido com e sem o uso de operações SIMD, o tempo de execução pode ser reduzido em até $37 \%$ e o consumo de energia em $35 \%$.

\footnotetext{
${ }^{1} \mathrm{O}$ presente trabalho teve financiamento parcial pela FAPERGS nos projetos 19/2551-0001224-1 e 19/2551-0001689-1, CNPq PIBIC e FAPERGS PROBIC.
} 


\section{Trabalhos Relacionados}

Os trabalhos propostos por [Inoue 2016], [Ponte et al. 2017], puderam demonstrar que ao se fazer uso de instruções do tipo SIMD, há significativas reduções de load e store. Além do mais, em [Ponte et al. 2017], demonstrou-se que há ganhos de desempenho de até 6.3 vezes ao se utilizar as diretivas OpenMP SIMD em comparação a autovetorização realizada apenas pelo compilador.

Em [Abbo 2004], utilizando processadores Xetal SIMD e os escalando (adicionando unidades de processamento), foi demonstrado que com o uso do paralelismo é possível reduzir gastos de energia. Ainda assim, a redução é intrínseca com a complexidade dos algoritmos, pois com um número específico de unidades de processadores a diferença se estabiliza.

Já em [Jakobs and Rünger 2018], cinco implementações de um mesmo algoritmo (eliminação Gaussiana) foram executadas em três processadores Intel Core i7 de especificações similares mas arquiteturas diferentes. Observou-se que cada processador se distingue pelas diferentes (porém semanticamente parecidas) instruções para stores e loads da API AVX (Um conjunto de instruções SIMD da Intel). Eles demonstraram que inclusive entre as próprias instruções SIMD com as condições certas, pode ser reduzido o tempo de execução e o consumo de energia de aplicações.

O trabalho tem como diferencial o fato de realizar os testes a fim de comparar o uso ou não de instruções vetoriais. Além disso foi levado em consideração tanto o desempenho quanto à eficiência energética.

\section{Metodologia}

Quatro aplicações com diferentes características foram selecionadas: CFD Solver, consiste em um solver de volume finito para grids não estruturadas para equações tridimensionais de Euler [Che et al. 2009]. Hotspot, é uma aplicação baseada em uma grid estruturada, utilizada para estimar a temperatura do processador com base no floorplan da arquitetura e em medidas de consumo de potência [Che et al. 2009]. Método de Jacobi, um algoritmo iterativo para determinar a solução de sistemas lineares envolvendo uma grande porcentagem de coeficientes zero. Decomposição LU (LUD), que consiste de um algoritmo para calcular as soluções de um conjunto de equações lineares, seu kernel decompõe uma matriz em um produto entre uma matriz triangular superior e uma inferior [Che et al. 2009]. De maneira geral estes testes são realizados utilizando vetores de tipos numéricos para adquirir os dados do profile.

Os experimentos foram realizados em um ambiente com processador AMD Ryzen 9 3900x contendo 12 núcleos e 24 threads com 32GB de memória e com Sistema Operacional Ubuntu. O compilador utilizado foi o GCC 9.2.0 com flags de otimização - $O 3$ e -fopenmp-simd (aplicações que exploram SIMD). Cada aplicação foi executada 10 vezes com diferentes números de threads: 1, 2, 4, 6, 8, 10, 12, 24 com o governor do DVFS (Dynamic voltage and frequency) mantido em ondemand e com as threads OpenMP pinadas, a fim de não superaquecer o processador e seus componentes.

\section{Resultados Experimentais}

As Figuras 1 e 2 apresentam os resultados de desempenho e consumo de energia para as quatro aplicações em função do número de threads. De uma maneira geral, pode- 


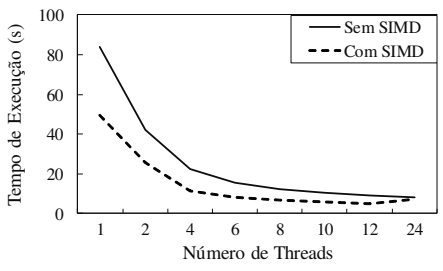

(a) Hotspot

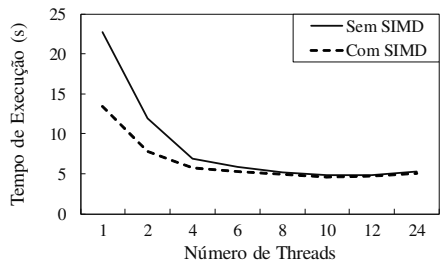

(c) LUD

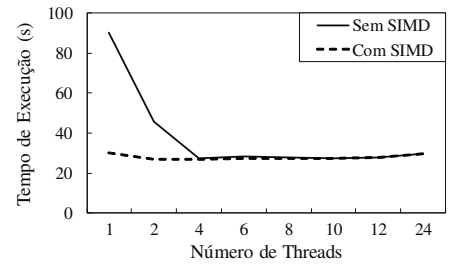

(b) Jacobi

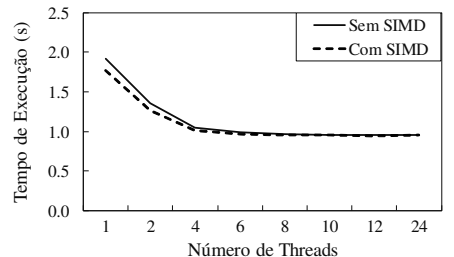

(d) CFD

Figura 1. Tempo de execução das aplicações sob diferentes números de threads

mos observar que a exploração de operações SIMD proporcionaram um melhor desempenho e menor consumo de energia, quando comparado a execução das aplicações sem operações SIMD, na maioria dos casos. É possível notar na Figura 2.a que se pode melhorar em $41.17 \%$ do valor total quando utilizando um único thread. Se mantém um $\Delta t$ (i.e. variação de tempo) relevante, porém se reduz a partir da utilização de $\mathbf{4}$ threads enquanto que a partir dos 12 threads a diferença se reduz a um nível desprezível. Já nas Figuras 2.b e 2.c, percebe-se uma redução de $66.66 \%$ em Jacobi e $38.88 \%$ em LUD ambos quando executam com uma única thread. Possuem signficativo $\Delta$ t até 4 threads. Por fim, na Figura 2.d a utilização de SIMD mostra uma aceleração de $3.84 \%$ quando utilizando 1 thread, tendo algum diferencial até 4 threads, a partir disso deixa de ter um $\Delta \mathrm{t}$ visível.

Quando o consumo de energia é considerado, a diferença do uso de SIMD aumenta. Na Figura 3.a se mantém um diferencial de até $42.85 \%$ com 1 thread, sendo estável até a execução com 12 threads. Na Figura 3.b, há uma redução considerável na diferença do consumo de energia (64.27\% de com 1 thread) conforme o número de threads aumenta. Novamente, na Figura 3.c, o $\Delta W$ (i.e. diferença de energia) tem seu pico em $38.88 \%$ com 1 thread, tornando a se reduzir a partir de 4 threads. Por outro lado, na Figura 3.d, pode-se observar um ganho de eficiência energética acerca de $3.50 \%$ até 4 threads. Após isso, ocorre uma redução na diferença em até 8 threads, onde nota-se uma diferença negativa de $2.56 \%$, ou seja, torna-se ineficiente energeticamente, retornando a um $\Delta \mathrm{W}=0$ aos 12 threads.

É perceptível a redução, tanto de tempo quanto ao gasto de energia. Nas análises foi possível verificar que em todos os casos, até a execução com 4 threads o uso de operações SIMD traz ganhos pertinentes. Dentre os testes, o CFD possui o menor aproveitamento das operações SIMD, devido a sua natureza. Em contrapartida, no Hotspot é possível ver resultados estáveis independente do número de threads sendo utilizado. 


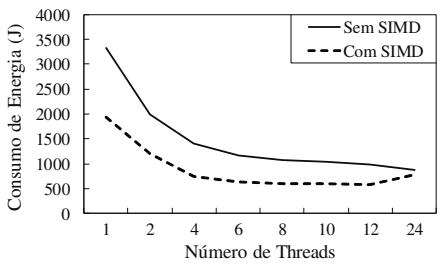

(a) Hotspot

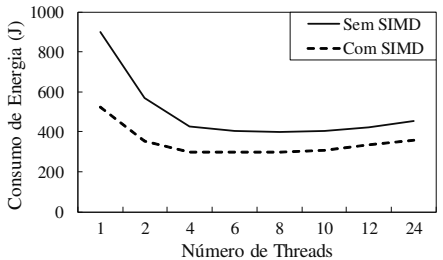

(c) LUD

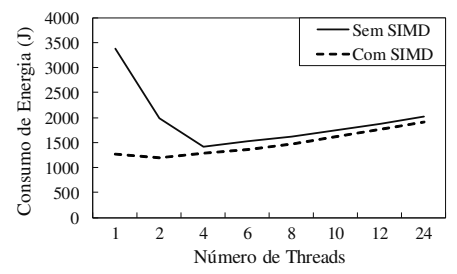

(b) Jacobi

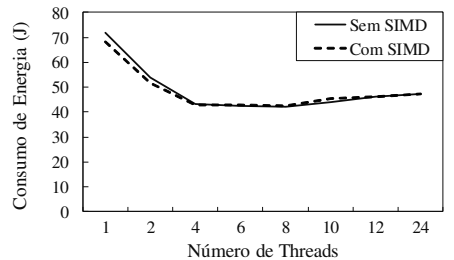

(d) CFD

Figura 2. Consumo de energia das aplicações sob diferentes números de threads

\section{Conclusão}

Com base nos resultados apresentados, é possível notar que a utilização dos registros vetoriais pode resultar em uma redução de energia significativa e uma melhoria no desempenho de determinadas aplicações. Entretanto, existem situações específicas onde o uso das operações SIMD tornam a aplicação ineficiente, tanto no quesito de consumo de energia quanto no tempo de execução, sendo necessário uma análise do projeto de software visando os prós e contras, a fim de tomar uma decisão a adotar ou não o uso de operações vetoriais.

\section{Referências}

Abbo, A. A. e. a. (2004). Power consumption of performance-scaled simd processors. In Integrated Circuit and System Design. Power and Timing Modeling, Optimization and Simulation, pages 532-540. Springer Berlin Heidelberg.

Chapman, B., Jost, G., and Pas, R. V. D. (2007). Using OpenMP - Portable Shared Memory Programming.

Che, S., Boyer, M., Meng, J., Tarjan, D., Sheaffer, J. W., Lee, S.-H., and Skadron, K. (2009). Rodinia: A benchmark suite for heterogeneous computing. In IEEE Int. Symp. on Workload Characterization, pages 44-54, DC, USA. IEEE Computer Society.

Inoue, H. (2016). How simd width affects energy efficiency: A case study on sorting. In COOL CHIPS XIX, pages 1-3.

Jakobs, T. and Rünger, G. (2018). On the energy consumption of load/store avx instructions. In FedCSIS, pages 319-327.

Ponte, C., González-Domínguez, J., and Martín, M. J. (2017). Evaluation of openmp simd directives on xeon phi coprocessors. In HPCS, pages 389-395. 